\title{
Insatisfação com a imagem corporal e fatores associados em adolescentes
}

\author{
Body image dissatisfaction and associated factors in adolescents
}

Giulia Xavier de Carvalho (https://orcid.org/0000-0001-7002-4479) ${ }^{1}$

Ana Paula Nogueira Nunes (https://orcid.org/0000-0002-1825-6990) ${ }^{2}$

Claudia Leite Moraes (https://orcid.org/0000-0002-3223-1634) ${ }^{3}$

Gloria Valeria da Veiga (https://orcid.org/0000-0002-7985-0213) ${ }^{1}$
${ }^{1}$ Instituto de Nutrição Josué de Castro, Universidade Federal do Rio de Janeiro. Av. Carlos Chagas Filho 373, Cidade Universitária. 21941-902 Rio de Janeiro RJ Brasil.

giuliaxc.1@gmail.com

${ }^{2}$ Universidade Federal dos

Vales do Jequitinhonha e

Mucuri. Diamantina MG

Brasil.

${ }^{3}$ Instituto de Medicina

Social, Universidade do

Estado do Rio de Janeiro.

Rio de Janeiro RJ Brasil.

\begin{abstract}
This study aimed to examine the factors associated with body dissatisfaction in adolescents. This is a cross-sectional study with students from two public and four private schools in the metropolitan region of Rio de Janeiro. Body dissatisfaction was evaluated using the Body Silhouette Scale, by the difference between the image they perceive as their current image and the one they would like to have. The association was assessed by hierarchical logistic regression multinomial model, by estimating odds ratios (OR) and 95\% confidence intervals (95\%CI). Among the 1,019 adolescents evaluated (13-19 years), 75\% showed body dissatisfaction, $41.4 \%$ wishing for a smaller silhouette and 33.7\% wishing for larger silhouettes. The wish for a smaller silhouette was more significant in girls, overweight adolescents, those with an unsatisfactory meal pattern, and with higher waist circumference. Adolescents that had been exposed to teasing due to their body shape increased the probability of wishing for both smaller and larger silhouettes. Strategies are required for a more positive perception of body image, especially for girls and for overweight adolescents, which provide guidance on adequate meal consumption and prevent exposure to peer teasing, valuing coexistence and well-being in the face of existing bodily differences.
\end{abstract}

Key words Body image, Adolescents, Overweight
Resumo $O$ objetivo do estudo foi examinar os fatores associados a insatisfação com a imagem corporal (IIC) em adolescentes. Estudo transversal com estudantes de duas escolas públicas e quatro escolas privadas da região metropolitana do Rio de Janeiro. A IIC foi avaliada pela Escala de Silhuetas Corporais, pela diferença entre a imagem que percebem como a sua atual e a que gostariam de ter. A associação foi investigada por meio de regressão logística multinomial hierarquizada, estimando-se as odds ratios (OR) e intervalos de confiança de 95\% (IC95\%). Entre os 1.019 adolescentes avaliados (13 a 19 anos), 75,0\% apresentaram IIC, 41,4\% desejando ter silhueta menor e $33,7 \%$ desejando ter silhueta maior do que a auto percebida. O desejo de ter uma silhueta menor foi maior nas meninas, naqueles que tinham padrão de refeição insatisfatório, com excesso de peso e perímetro da cintura elevado. A exposição a provocações por causa do corpo aumentou a chance para desejar silhuetas menores e maiores. Evidencia-se a necessidade de estratégias para uma percepção mais positiva da imagem corporal, principalmente para meninas e jovens com excesso de peso, que orientem sobre consumo adequado de refeições e para prevenção de exposição a provocações pelos pares, valorizando a convivência e o bem estar frente às diferenças corporais existentes. Palavras-chave Imagem corporal, Adolescentes, Sobrepeso 


\section{Introdução}

A imagem corporal faz parte do mecanismo de identidade pessoal que tem uma construção multidimensional ${ }^{1}$. Na sociedade contemporânea ocidental é comum observar-se muitas pessoas que tentam se enquadrar nos padrões de beleza considerados ideais, gerando insatisfação com a imagem corporal quando não bem sucedidas². Tal insatisfação é mais evidente nos adolescentes por serem mais vulneráveis as pressões impostas pela sociedade, familiares, amigos e pela mídia para que alcancem o corpo perfeito como forma de obtenção de aceitação e sucesso social ${ }^{3}$.

$\mathrm{Na}$ busca pelos ideais estéticos impostos, muitos adolescentes recorrem a dietas muito restritivas, ao exercício físico exagerado, ao uso de diuréticos e laxantes, de anabolizantes, assim como a procura por cirurgias plásticas estéticas desnecessárias ${ }^{4}$. Tais comportamentos podem estar na gênese de transtornos alimentares como a anorexia nervosa, a bulimia e a compulsão alimentar, que podem comprometer, gravemente, a saúde dos jovens.

A insatisfação com a imagem corporal tem sido relacionada a alguns fatores demográficos como ser adolescente e do sexo feminino ${ }^{5}$, psicossociais como estar envolvido com exposição ao bullying ${ }^{6}$ relacionados aos hábitos de vida como inatividade física e práticas alimentares irregulares $^{7-9}$ e estado nutricional como apresentar excesso de peso $^{5,10}$. Todavia, a interrelação entre estes fatores exige que sejam analisados em um contexto multidimensional e numa perspectiva hierárquica que permita a identificação daqueles que se associam ao desfecho de forma independente.

Sendo assim, o presente estudo pretende avaliar a magnitude da insatisfação com a imagem corporal em adolescentes e identificar subgrupos de maior risco, com base na análise de possíveis fatores associados. Tal investigação poderá ser útil para subsidiar a discussão sobre o problema e implementação de estratégias que promovam uma imagem corporal mais positiva entre adolescentes evitando, consequentemente, efeitos adversos à saúde provocados por tal insatisfação.

\section{Métodos}

\section{População avaliada}

Foram analisados os dados de adolescentes matriculados no $1^{\circ}$ ano do Ensino Médio, em duas escolas públicas e quatro escolas privadas da região metropolitana do Rio de Janeiro, em 2010, que fizeram parte da linha de base do Estudo Longitudinal de Avaliação Nutricional de Adolescentes (ELANA).

Os critérios de exclusão para fazer parte do estudo principal foram: ser portadores de deficiência física que impedisse a avaliação antropométrica, adolescentes grávidas e aqueles que estavam sendo submetidos a algum tratamento medicamentoso para controle de peso. Foram identificados 1.131 participantes elegíveis para o estudo. Destes, 92 não participaram devido a: não apresentarem autorização dos pais e/ou responsáveis (17), por se recusarem a participar (66) ou por terem faltado no dia da coleta de dados (9). Especificamente para este estudo, foram excluídos 20 adolescentes que não apresentaram dados relativos à percepção da imagem corporal, totalizando 1.019 adolescentes para serem analisados, correspondendo a taxa de resposta de $90 \%$.

\section{Coleta de dados e variáveis do estudo}

A coleta de dados foi realizada entre fevereiro a agosto de 2010, por equipe devidamente treinada, mediante autorização dos pais e/ou responsáveis e pelo assentimento dos alunos.

As informações foram coletadas a partir da aplicação de questionários auto preenchidos para investigação da variável de desfecho (insatisfação com a imagem corporal) e das variáveis de exposição: demográficas (sexo, idade, cor/raça), socioeconômicas (classe econômica, escolaridade do chefe da família e tipo de escola), psicossocial (exposição ao teasing), relacionadas ao estilo de vida (padrão de refeições, tabagismo, experimentação e consumo atual de álcool, prática de atividade física e comportamento sedentário), antropométricas e de composição corporal (índice de massa corporal e percentual de gordura corporal).

Para a avaliação da percepção da imagem corporal, aplicou-se a Escala de Silhuetas Corporais adaptada para adolescentes ${ }^{11}$. Esta é composta por nove silhuetas para as meninas e nove para os meninos, que variavam desde a figura correspondente à extrema magreza $\left(\mathrm{n}^{\circ} 1\right)$ até à obesidade $\left(n^{\circ} 9\right)^{12}$. A escala foi acompanhada das seguintes perguntas: 1. Marque a figura com a qual você acha que mais se parece; 2 . Marque a figura com a qual você mais gostaria de parecer. A variável "insatisfação com a imagem corporal" foi definida por meio da diferença entre a pontuação correspondente à silhueta que o adolescente julgava 
ser a sua atual (pergunta 1) e a que gostaria de se parecer (pergunta 2), podendo variar de -8 a +8 . A pontuação negativa indicava desejo de ter silhuetas maiores e a pontuação positiva desejo de ter silhuetas menores.

A informação sobre cor/raça foi obtida por auto avaliação. Os adolescentes foram questionados sobre como se classificavam, e tinham, como opção de resposta, as mesmas opções investigadas pelo Censo do Instituto Brasileiro de Geografia e Estatísticas ${ }^{13}$ preta, parda, branca, amarela ou indígena. Nas análises de associação utilizou-se as categorias branco / não branco, agrupando-se as classes de cor da pele preta, parda, amarela e indígena, assim como em outros estudos realizados no Brasil ${ }^{14}$ devido à baixa frequência de amarelos $(2,8 \%)$ e indígenas $(2,1 \%)$ e pela possibilidade de que indivíduos pretos ou pardos possam se auto classificar da mesma forma.

A idade foi calculada pela diferença entre a data de nascimento (obtida nas fichas de matrícula dos adolescentes) e a data de avaliação, por considerar-se esta fonte mais fidedigna do que a informação a partir do próprio adolescente.

A classe econômica foi avaliada com base no Critério de Classificação Econômica Brasil 2008 (CCEB), aprovado pela ABA/ABIPEME (Associação Brasileira de Anunciantes/Associação Brasileira dos Institutos de Mercado ${ }^{15}$ o qual utiliza o sistema de atribuição de pontos à quantidade de bens duráveis; presença de banheiro na residência; existência de empregado doméstico mensalista no domicílio e a escolaridade do chefe do domicílio. A classe econômica é classificada em A, B, C, D e E, sendo a categoria A de maior poder e $\mathrm{E}$ a de menor poder de compra, de acordo com a pontuação na escala. Nas análises de associação, foram consideradas as categorias A, B e C/D agrupadas, em decorrência de pequenas frequências e até ausência nas categorias mais baixas (E).

A escolaridade do chefe de família foi informada pelos pais ou responsáveis dos adolescentes, por meio de questionário enviado para o domicílio ou por entrevista por telefone, com base na pergunta "Qual foi a última série (ou ano) e o último grau concluídos na escola pela mãe e pelo pai do adolescente?", tendo também a opção de resposta "nunca estudou".

A frequência de refeições foi investigada com base em perguntas sobre frequência do consumo de desjejum, almoço, jantar, com as seguintes opções de resposta para cada uma destas refeições: diariamente, 5 a 6 vezes por semana, 3 a 4 vezes por semana, 1 a 2 vezes por semana e nunca ou quase nunca. Para classificar o adolescente quanto a frequência no consumo de refeições, foi utilizado score, que variou de 0 a 9 pontos, resultante da soma dos pontos referentes à frequência de consumo de desjejum, almoço e jantar. Caso o adolescente apresentasse um consumo diário de cada uma destas refeições ganhava 0 ponto; 3 a 6 vezes na semana $=1$ ponto; 1 ou 2 vezes na semana $=2$ pontos e nunca ou quase nunca $=3$ pontos. Foi considerado padrão de refeição satisfatório quando a soma dos pontos variou de 0 (ou seja, consumo diário das três refeições) a 1 (até 3 a 6 vezes por semana em, no máximo, uma das três refeições), segundo critério proposto por Estima et al. ${ }^{16}$.

A experimentação no consumo de álcool e o consumo atual nos últimos trinta dias foram investigados com base nas respectivas perguntas: "Alguma vez na vida, você já experimentou bebida alcoólica?", "Nos últimos 30 dias, em quantos dias você tomou, pelo menos, um copo ou uma dose de bebida alcoólica?". Foi considerado presença de consumo de álcool quando o adolescente informou consumo em, pelo menos, 1 dia nos últimos trinta dias. Para avaliação da prática de tabagismo, os adolescentes responderam à pergunta "Atualmente, você fuma cigarros?".

A prática de atividade física foi avaliada por meio da versão curta do Questionário Internacional de Atividade Física (International Physical Activity Questionnaire - IPAQ), validado no Brasil para adolescentes ${ }^{17}$. Com base neste questionário os adolescentes são classificados como muito ativos, ativos, moderadamente ou irregularmente ativos e sedentários ${ }^{18}$. No presente estudo, em decorrência de frequências pequenas em algumas categorias, os adolescentes foram classificados em três grupos: muito ativos ou ativos; irregularmente ativos e pouco ativos.

O comportamento sedentário foi avaliado pela frequência do hábito de assistir TV e uso de computador ou videogame, com base nas seguintes perguntas: "Você assiste televisão?", "Você usa computador ou joga vídeo game?". Cada uma das perguntas teve como opção as seguintes respostas: nunca ou quase nunca, 1 a 2 vezes por semana, 3 a 4 vezes por semana, 5 a 6 vezes por semana, diariamente. Questionou-se ainda "Geralmente, quantas horas por dia você assiste televisão?", "Geralmente, quantas horas por dia você usa computador ou vídeo game?”. O total de horas por dia gasto em cada uma destas atividades foi calculado multiplicando o número de horas por dia informado pela frequência semanal, dividido por 7 dias. A presença de comportamento sedentário foi considerada quando o tempo diá- 
rio com quaisquer uma destas práticas era igual ou superior a 2 horas $^{19}$.

A exposição ao teasing (ou provocação) foi investigada com base no instrumento Child-Adolescent Teasing Scale (CATS $)^{20}$. Neste estudo foi utilizada apenas a subescala relacionada ao corpo e peso, pois estudos anteriores indicaram associação de teasing corporal com atitudes negativas para a saúde do adolescente, tais como, alimentação restritiva, comportamentos bulímicos, baixa autoestima, internalização do ideal da magreza e depressão, além de uma ligação causal com insatisfação com a imagem corporal $^{21,22}$. Esta subescala é composta por duas perguntas: 1) "Com que frequência zoam/caçoam de mim por causa do meu corpo"; 2) "Com que frequência zoam/ caçoam de mim por causa do meu peso", com as seguintes opções de resposta: nunca ( $=0$ ponto); às vezes ( $=1$ ponto); quase sempre ( $=2$ pontos); sempre ( $=3$ pontos). Cada item é acompanhado por uma questão sobre o quanto o ato aborrece o adolescente quando este é praticado, com as mesmas opções de resposta. Para o presente estudo, foi criado um score contínuo formado a partir da soma da pontuação em cada um dos itens da subescala, multiplicado pela pontuação correspondente para a frequência com que se sentiam aborrecidos pela provocação, podendo variar de 0 a 18 pontos. Considerou-se exposição ao teasing qualquer score diferente de zero, ou seja, qualquer "frequência de aborrecimento" para uma dada "frequência de provocação".

Para aferição das medidas antropométricas os avaliadores foram padronizados com base nos critérios de Habicht ${ }^{23}$. Foram aferidas as seguintes medidas: peso, estatura e perímetro da cintura. O peso foi medido em balança eletrônica e portátil com capacidade de até $180 \mathrm{~kg}$ e variação de 50g. A estatura foi medida por meio de um antropômetro portátil, Alturexata ${ }^{\oplus}$, com amplitude de 0 a $213 \mathrm{~cm}$ e variação de $0,1 \mathrm{~cm}$. Foram realizadas duas medidas de estatura e calculada a média aritmética, admitindo-se uma variação máxima de $0,5 \mathrm{~cm}$ entre as duas medidas. Para aferição do peso e da estatura os adolescentes estavam descalços, sem acessórios e vestindo roupas leves. Com os dados de peso e da estatura calculou-se o Índice de Massa Corporal (IMC = peso em kg/ estatura $^{2}$ em metros). A classificação quanto à adequação do peso foi feita com base nos pontos de corte do IMC, específicos por idade e sexo, segundo critérios da Organização Mundial da $S_{\text {Saúde }}{ }^{24}$. Os adolescentes foram classificados em baixo peso (IMC $<-2 \mathrm{Z}$ escore), peso adequado (IMC $\geq-2$ e $<1 \mathrm{Z}$ escore), excesso de peso
(IMC $\geq 1$ e $<2 \mathrm{Z}$ escore) e obesidade (IMC $\geq$ $2 \mathrm{Z}$ escore). Nas análises de associação os adolescentes foram classificados em: com excesso de peso (agrupando-se excesso de peso e obesidade) e sem excesso de peso (agrupando-se baixo peso e peso adequado).

O perímetro da cintura foi medido na menor extensão horizontal do tronco, com fita métrica inextensível de $150 \mathrm{~cm}$ e variação de $0,1 \mathrm{~cm}$. Foram realizadas duas medidas e calculada a média aritmética, admitindo-se uma variação máxima de $1,0 \mathrm{~cm}$ entre elas. Os adolescentes foram classificados como tendo perímetro da cintura elevado quando este estava acima do percentil 90 da distribuição da própria amostra.

A composição corporal foi estimada pelo método da bioimpedância elétrica, com analisador de gordura corporal tetrapolar (RJL System modelo $\left.101 Q^{\circ}\right)$. Para esta avaliação os adolescentes foram orientados a seguir um protocolo para controle sobre a ingestão de líquidos, café, bebidas alcoólicas, e uso de medicamentos laxativos ou diuréticos. A massa livre de gordura (MLG) foi estimada com base na equação validada para adolescentes ${ }^{25}$, conforme segue: $\mathrm{MLG}=0,61 \mathrm{RI}+$ $0,25 \mathrm{MC}+1,31$. Onde: $\mathrm{RI}=$ Estatura $^{2}(\mathrm{~cm}) /$ Resistência $(\Omega) ; \mathrm{MC}=$ Massa corporal ( peso em $\mathrm{kg}$ ).

A gordura corporal foi estimada pelo cálculo da diferença entre a massa corporal $(\mathrm{kg})$ e MLG $(\mathrm{kg})$, obtendo-se depois a gordura corporal relativa (\%GC). Foram considerados com \%GC elevado os adolescentes que apresentaram valores superiores a $25 \%$ para os meninos e $30 \%$ para as meninas ${ }^{26}$.

\section{Análise dos dados}

\section{Imputação múltipla de dados}

Devido a ocorrência de dados faltantes, sobretudo nas variáveis socioeconômicas (aproximadamente 20\%), optou-se por realizar a imputação múltipla para dados faltantes utilizando-se o comando Multiple Imputation do software Statistical Program for the Social Sciences, versão 19,0 (SPSS, Chicago, IL). Para tal procedimento assumiu-se características de perdas aleatórias, o que permite que a imputação de dados possa ser feita a partir de outras variáveis disponíveis no banco de dados ${ }^{27}$. Optou-se pela realização de 10 bancos imputados previstos por regressão linear, inserindo todas as variáveis analisadas no presente estudo. Utilizou-se o método MCMC (Markov Chain Monte Carlo) para padrão de perdas arbitrário (monotônico ou não monotônicos) e 20 iterações para os bancos imputados ${ }^{27,28}$. 
Nas análises estatísticas, realizaram-se análises bivariadas e multivariadas, por meio de regressão logística multinomial, para investigar a associação entre as variáveis independentes e a insatisfação com a imagem corporal, estimandose $a$ odds ratio (OR) e intervalo de confiança de 95\% (IC95\%). A variável resposta (insatisfação com a imagem corporal), foi analisada em três categorias: (1) satisfeito (referência), (2) desejo de ter silhuetas maiores e (3) desejo de ter silhuetas menores. Todas as variáveis independentes investigadas nas análises bivariadas fizeram parte das análises multivariadas, com o objetivo de se observar a interdependência entre elas na associação com o desfecho, mesmo que não tenham mostrado associação com o mesmo na análise bivariada.

A análise multivariada foi feita com base em modelo operacional hierarquizado, esquematizado na Figura 1. Com base neste modelo, hipotetiza-se que a relação das variáveis de exposição investigadas com o desfecho insatisfação corporal se dá por determinações mais distais como as esperadas para as variáveis demográficas e socioeconômicas, passando pelas dimensões relacionadas ao estilo de vida e aspectos psicossociais relacionados ao adolescente até as dimensões mais proximais relacionadas aos aspectos individuais do adolescente como as variáveis antropométricas e de composição corporal. Esta forma de análise foi baseada em Victora et al. ${ }^{29}$. Assim, a primeira dimensão do modelo contempla as variáveis demográficas e socioeconômicas: sexo (masculino e feminino), faixa etária $(<15$ anos e $\geq 15$ anos), cor (branco e preto/pardo/amare- lo/indígena), tipo de escola (pública ou privada), classificação econômica (A, B, C/D agrupadas), e escolaridade do chefe da família (nenhuma ou até 4 anos de estudo, 5 a 8 anos de estudo, 9 a 12 anos de estudo); a segunda dimensão envolve a questão psicossocial, aqui representada pela variável exposição ao teasing associada ao corpo ( riáveis relacionadas ao estilo de vida que foram: padrão de refeição (insatisfatório e satisfatório), fumo (sim e não), experimentação de bebida alcoólica (sim e não), consumo atual de bebida alcóolica (sim e não), prática de atividade física (a pouco ativos, b - irregularmente ativos, $\mathrm{c}$ - ativos ou muito ativos) e comportamento sedentário ( sim e não). A quarta e última dimensão envolve as características antropométricas e de composição corporal do adolescente: classificação com base no IMC (com excesso de peso e sem excesso de peso), perímetro da cintura ( $<\mathrm{P} 90$ e $\geq \mathrm{P} 90$ da distribuição da amostra investigada) e percentual de gordura corporal (elevado e não elevado). As variáveis foram introduzidas no modelo em bloco, seguindo-se a ordem da dimensão mais distal para a mais proximal ao desfecho. As variáveis que apresentaram nível de significância de $\mathrm{p}$ $<0,05$ foram mantidas em cada etapa da análise hierárquica, analisadas na etapa seguinte e retidas no modelo final.

As análises estatísticas e de imputação múltipla de dados foram realizadas utilizando-se o software Statistical Program for the Social Sciences, versão 19.0 (SPSS - IL, CHICAGO, 2009).

A pesquisa ELANA, cujos dados foram analisados neste estudo, foi aprovada pelo Comitê de

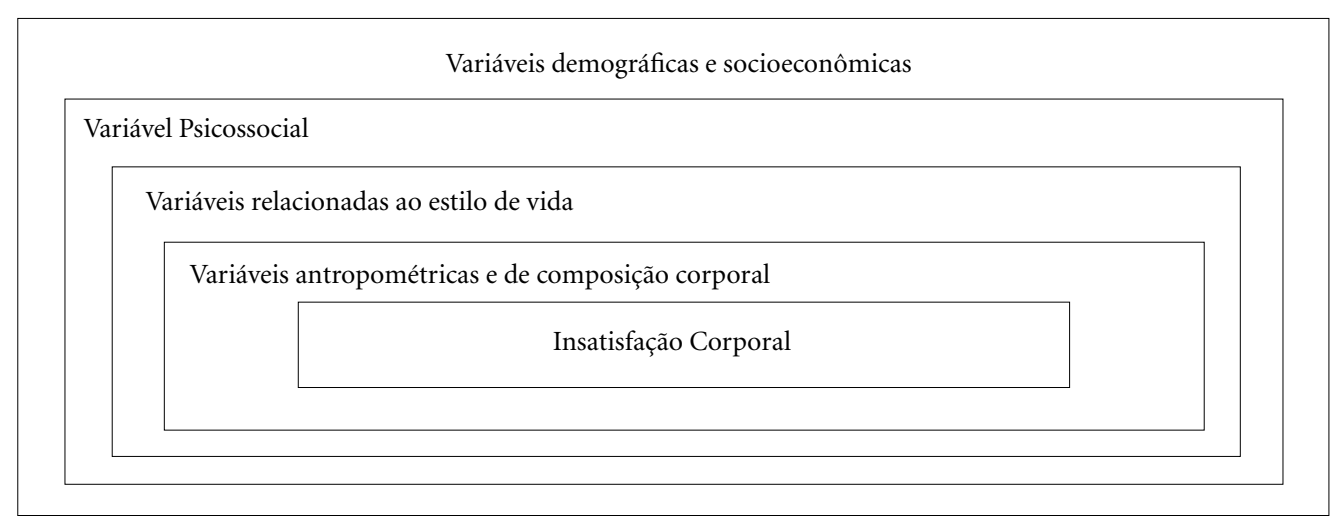

Figura 1. Modelo operacional hierarquizado para a análise multivariada. 
Ética em Pesquisa do Instituto de Medicina Social da Universidade Estadual do Rio de Janeiro. Só participaram do estudo os adolescentes que quiseram e que tiveram o Termo de Consentimento Livre e Esclarecido assinado pelos pais ou responsáveis.

\section{Resultados}

O perfil da amostra é descrito na Tabela 1 . Verifica-se que $53,4 \%$ dos adolescentes era do sexo feminino e $53,8 \%$ de cor preta/parda/amarela ou indígena; a maioria estava na faixa etária acima de 15 anos $(81,8 \%)$ e com escolaridade do chefe da família entre 9 a 12 anos $(69,6 \%)$; pouco mais da metade era da classe social B $(53,2 \%)$ e cerca de um quarto dos adolescentes relataram já ter sofrido exposição ao teasing; pouco mais da metade $(54,6 \%)$ tinham um padrão de refeições insatisfatório, a grande maioria nunca havia fumado $(97,3 \%)$ mas já haviam experimentado álcool (71,6\%), embora 63,1\% informaram não ter consumo atual de bebidas alcoólicas. Com base na aplicação do IPAQ, a maioria dos adolescentes $(75,5 \%)$ foram classificados como ativos ou muito ativos, embora $71,3 \%$ tenham relatado hábito sedentário de mais de 2 horas assistindo TV ou vídeo game. Na classificação com base no IMC, $26,2 \%$ apresentavam excesso de peso, mas apenas $5,3 \%$ apresentavam percentual de gordura elevado.

Entre os adolescentes avaliados, $422(41,4 \%)$ desejavam uma silhueta menor e $343(33,7 \%)$ desejavam uma silhueta maior, ou seja, $765(75,1 \%)$ estavam insatisfeitos e informaram desejar ter uma silhueta diferente daquela na qual se auto percebiam.

Com base na análise bivariada observou-se que o desejo de ter silhuetas menores do que aquelas com as quais se identificavam se apresentou em maior chance nas meninas do que nos meninos (OR = 1,97, IC95\% 1,44-2,71), nos adolescentes com excesso de peso do que naqueles sem excesso (OR $=6,22$, IC95\% 4,18-9,26), com perímetro da cintura $\geq$ percentil 90 do que naqueles com valor abaixo deste limite $(\mathrm{OR}=15,70$, IC95\% 5,95-41,6), com o \% de gordura corporal elevado do que com valores adequados $(\mathrm{OR}=$ 9,53, IC95\% 2,44-37,2), naqueles que apresentaram padrão de refeições insatisfatório do que com padrão satisfatório ( $\mathrm{OR}=2,70$, IC95\% 1,96$3,74)$. Ser exposto ao teasing aumentou a chance, tanto para o desejo de ter silhuetas menores (OR $=4,79$, IC95\% 3,00-7,65) quanto de ter silhuetas
Tabela 1. Caracterização dos adolescentes segundo categorias das variáveis investigadas.

\begin{tabular}{|c|c|c|}
\hline Variáveis (N) & $\%$ & IC95\% \\
\hline \multicolumn{3}{|l|}{ Sexo (1.019) } \\
\hline Feminino & 53,4 & $50,3-56,5$ \\
\hline Masculino & 46,6 & $43,5-49,7$ \\
\hline \multicolumn{3}{|l|}{ Faixa etária (1.019) } \\
\hline$\geq 15$ anos & 81,8 & $79,5-84,2$ \\
\hline$<15$ anos & 18,2 & $15,8-20,5$ \\
\hline \multicolumn{3}{|l|}{ Cor (1.017) } \\
\hline Não branco & 53,8 & $50,7-56,9$ \\
\hline Branco & 46,2 & $43,1-49,3$ \\
\hline \multicolumn{3}{|l|}{ Tipo de escola (1.019) } \\
\hline Privada & 49,8 & $46,7-52,8$ \\
\hline Pública & 50,2 & $47,2-53,3$ \\
\hline \multicolumn{3}{|c|}{ Classificação econômica (810) } \\
\hline A1 & 2,0 & $1,0-2,9$ \\
\hline $\mathrm{A} 2$ & 13,7 & $11,3-16,1$ \\
\hline B1 & 23,6 & $20,7-26,5$ \\
\hline B2 & 29,6 & $26,5-32,8$ \\
\hline $\mathrm{C} 1$ & 22,7 & $19,8-25,6$ \\
\hline $\mathrm{C} 2$ & 7,0 & $5,3-8,8$ \\
\hline $\mathrm{D}$ & 1,4 & $0,6-2,2$ \\
\hline \multicolumn{3}{|l|}{$\begin{array}{l}\text { Escolaridade do chefe de } \\
\text { família (830) }\end{array}$} \\
\hline 9 a 12 anos & 69,6 & $66,5-72,8$ \\
\hline 5 a 8 anos & 19,5 & $16,8-22,2$ \\
\hline 0 a 4 anos & 10,8 & $8,8-13,0$ \\
\hline \multicolumn{3}{|l|}{ Teasing (1.014) } \\
\hline $\operatorname{Sim}$ & 24,2 & $21,5-26,8$ \\
\hline Não & 75,8 & $73,2-78,5$ \\
\hline \multicolumn{3}{|c|}{ Padrão de refeição (1.013) } \\
\hline Insatisfatório & 54,6 & $51,5-57,7$ \\
\hline Satisfatório & 45,4 & $42,3-48,5$ \\
\hline
\end{tabular}

continua

maiores $(\mathrm{OR}=2,98$, IC95\% 1,83-4,86) (Tabela 2).

Com base na análise multivariada hierarquizada (Tabela 3), observa-se que apenas as variáveis sexo e tipo de escola mostraram-se associadas com a insatisfação corporal na primeira etapa da análise, mantendo-se a mesma associação observada na análise bivariada para as meninas comparadas aos meninos e os alunos das escolas privadas apresentaram maior chance de desejar ter silhuetas menores comparados aos de escola pública.

Na segunda etapa, além do sexo e tipo de escola, foi inserida a variável psicossocial exposição ao teasing relacionado ao corpo, que se mostrou 
Tabela 1. Caracterização dos adolescentes segundo categorias das variáveis investigadas.

\begin{tabular}{|c|c|c|}
\hline Variáveis (N) & $\%$ & IC95\% \\
\hline \multicolumn{3}{|l|}{ Fumo (1.018) } \\
\hline Sim & 2,8 & $1,7-3,8$ \\
\hline Não & 97,2 & $96,2-98,3$ \\
\hline \multicolumn{3}{|l|}{ Álcool } \\
\hline \multicolumn{3}{|l|}{ Experimentação (1.019) } \\
\hline Sim & 71,6 & $68,9-74,4$ \\
\hline Não & 28,4 & $25,6-31,1$ \\
\hline \multicolumn{3}{|l|}{ Consumo atual (1.009) } \\
\hline Sim & 36,8 & $33,8-39,7$ \\
\hline Não & 63,2 & $60,3-66,2$ \\
\hline \multicolumn{3}{|l|}{ Atividade física (1.007) } \\
\hline Sedentários & 3,9 & $2,7-5,1$ \\
\hline Irregularmente ativo B & 12,2 & $10,2-14,2$ \\
\hline Irregularmente ativo A & 8,3 & $6,6-10,1$ \\
\hline Ativos & 53,3 & $50,2-56,4$ \\
\hline Muito ativos & 22,2 & $19,7-24,8$ \\
\hline \multicolumn{3}{|l|}{$\begin{array}{l}\text { Comportamento sedentário } \\
(1.018)\end{array}$} \\
\hline $\operatorname{Sim}$ & 71,3 & $68,5-74,1$ \\
\hline Não & 28,7 & $25,9-31,5$ \\
\hline \multirow{2}{*}{\multicolumn{3}{|c|}{$\begin{array}{l}\text { Classificação com base no } \\
\text { IMC (996) }\end{array}$}} \\
\hline & & \\
\hline Baixo Peso & 1,5 & $0,7-2,3$ \\
\hline Peso Adequado & 72,3 & $69,5-75,1$ \\
\hline Sobrepeso & 18,0 & $15,6-20,4$ \\
\hline Obesidade & 8,2 & $6,5-9,9$ \\
\hline \multicolumn{3}{|l|}{ Perímetro da Cintura (995) } \\
\hline$\geq \mathrm{P} 90$ & 9,8 & $8,0-11,7$ \\
\hline$<\mathrm{P} 90$ & 90,2 & $88,3-92,0$ \\
\hline \multicolumn{3}{|l|}{$\%$ de $\mathrm{GC}^{\ddagger}(980)$} \\
\hline Elevado & 5,3 & $3,9-6,7$ \\
\hline Não elevado & 94,7 & $93,3-96,1$ \\
\hline
\end{tabular}

${ }^{\mathrm{t}}$ Gordura corporal. modo que e os adolescentes com padrão de refeição insatisfatório apresentaram maior chance de desejar silhuetas menores (OR $=2,50$, IC95\% $1,78-3,51)$ e os jovens que fumavam apresentaram menor chance para o desejo de ter silhuetas, tanto menores $(\mathrm{OR}=0,36$, IC95\% 0,14- 0,90) quanto maiores $(\mathrm{OR}=0,34$, IC95\% 0,12-0,96) (Tabela 2).

$\mathrm{Na}$ quarta e última etapa, além das variáveis que se mostraram associadas a algum tipo de insatisfação corporal nas etapas anteriores (sexo, sofrer teasing, padrão de refeição insatisfatório e o hábito de fumar) foram inseridas as variáveis antropométricas e de composição corporal. As variáveis sexo, exposição ao teasing relacionado ao corpo e padrão de refeição insatisfatório mantiveram as associações observadas nas etapas anteriores. Com relação às variáveis antropométricas, observou-se que os adolescentes com perímetro da cintura acima do percentil $90(\mathrm{OR}=$ 3,71 , IC95\% 1,03-13,42) e os adolescentes com excesso de peso corporal (OR $=5,08$, IC95\% 3,19-8,08) apresentaram maior chance de desejar silhuetas menores quando comparados aos adolescentes com o perímetro da cintura abaixo do percentil 90 e com aqueles sem excesso de peso, respectivamente. Nesta etapa, o hábito de fumar manteve associação (com significância estatística limítrofe $\mathrm{p}=0,06$ ), com a insatisfação corporal de modo que os adolescentes que fumavam mantiveram uma menor chance de desejar silhuetas menores (OR $=0,36$, IC95\% 0,13-0,98). No modelo final para desejo de ter silhuetas menores foram retidas as variáveis sexo, a exposição ao teasing relacionado ao corpo, o padrão de refeição insatisfatório, perímetro da cintura acima do percentil 90 e excesso de peso. Para desejo de ter silhuetas maiores foi retida apenas a variável teasing relacionado ao corpo (Tabela 3 ).

\section{Discussão}

Este estudo apresenta como um de seus principais resultados a elevada prevalência de insatisfação com a imagem corporal entre os adolescentes. Cerca de três quartos da amostra estudada referem desejar ter uma silhueta corporal diferente da qual se percebe. Este achado está de acordo com a tendência que vem sendo demonstrada de altas frequências de insatisfação com a imagem corporal entre adolescentes independentemente do método de avaliaçãa $0^{5,30-32}$.

Embora a prevalência de insatisfação com a imagem corporal tenha sido elevada em ambos 
Tabela 2. Análise de regressão multinominal bivariada (Odds ratio e intervalo de confiança de 95\%) para a associação entre insatisfação com a imagem corporal e variáveis demográficas e socioeconômicas, antropométricas e de composição corporal, psicossocial e de estilo de vida.

\begin{tabular}{|c|c|c|c|c|c|c|c|c|c|}
\hline \multirow{3}{*}{ Variáveis } & \multirow{3}{*}{$\begin{array}{l}\text { Satisfeitos } \\
(\%)\end{array}$} & \multicolumn{6}{|c|}{ Insatisfeitos } & \multirow[b]{3}{*}{ IC95\% } & \multirow[b]{3}{*}{$\begin{array}{l}\text { Valor } \\
\text { de p }\end{array}$} \\
\hline & & \multicolumn{3}{|c|}{$\begin{array}{c}\text { Desejam silhuetas } \\
\text { menores }\end{array}$} & \multicolumn{3}{|c|}{$\begin{array}{c}\text { Desejam silhuetas } \\
\text { maiores }\end{array}$} & & \\
\hline & & $\%$ & OR & IC95\% & $\begin{array}{l}\text { Valor } \\
\text { de p }\end{array}$ & $\%$ & OR & & \\
\hline \multicolumn{10}{|l|}{$\begin{array}{l}\text { I. Demográficas } \\
\text { socioeconômicas }\end{array}$} \\
\hline \multicolumn{10}{|l|}{ Sexo } \\
\hline Feminino & 22,6 & 50,4 & 1,97 & $1,44-2,71$ & $<0,01$ & 27,0 & 0,80 & $0,58-1,11$ & 0,18 \\
\hline Masculino & 27,6 & 31,1 & 1,0 & & & 41,3 & 1,0 & & \\
\hline \multicolumn{10}{|l|}{ Faixa etária } \\
\hline$\geq 15$ anos & 25,2 & 39,7 & 0,76 & $0,51-1,14$ & 0,18 & 35,1 & 1,23 & $0,79-1,91$ & 0,36 \\
\hline$<15$ anos & 23,8 & 49,2 & 1,0 & & & 27,0 & 1,0 & & \\
\hline \multicolumn{10}{|l|}{ Cor } \\
\hline $\begin{array}{l}\text { Preto/pardo/amarelo/ } \\
\text { indígena }\end{array}$ & 24,7 & 38,8 & 0,89 & $0,65-1,21$ & 0,45 & 36,5 & 1,22 & $0,88-1,69$ & 0,23 \\
\hline Branco & 25,1 & 44,5 & 1,0 & & & 30,4 & 1,0 & & \\
\hline \multicolumn{10}{|l|}{ Tipo de escola } \\
\hline Particular & 25,0 & 46,2 & 1,25 & $0,91-1,70$ & 0,17 & 28,8 & 0,74 & $0,54-1,03$ & 0,07 \\
\hline Pública & 24,8 & 36,7 & 1,0 & & & 38,5 & 1,0 & & \\
\hline \multicolumn{10}{|l|}{$\begin{array}{l}\text { Classificação social e } \\
\text { Econômica }\end{array}$} \\
\hline A & 21,0 & 47,8 & 1,29 & $0,75-2,21$ & 0,35 & 31,2 & 0,85 & $0,49-1,48$ & 0,56 \\
\hline $\mathrm{B}$ & 27,8 & 40,8 & 0,83 & $0,55-1,25$ & 0,37 & 31,4 & 0,64 & $0,43-0,97$ & 0,03 \\
\hline C e D & 22,1 & 39,1 & 1,0 & & & 38,8 & 1,0 & & \\
\hline \multicolumn{10}{|l|}{$\begin{array}{l}\text { Escolaridade do chefe de } \\
\text { família }\end{array}$} \\
\hline 9 a 12 anos & 27,1 & 40,0 & 0,75 & $0,45-1,26$ & 0,28 & 32,9 & 0,84 & $0,47-1,53$ & 0,57 \\
\hline 5 a 8 anos & 18,6 & 44,3 & 1,22 & $0,65-2,27$ & 0,54 & 37,1 & 1,39 & $0,69-2,77$ & 0,36 \\
\hline 0 a 4 anos & 22,8 & 44,5 & 1,0 & & & 32,7 & 1,0 & & \\
\hline
\end{tabular}

os sexos, o perfil de insatisfação é oposto entre meninas e meninos. Enquanto as meninas apresentaram maior desejo por silhuetas menores, os meninos, ao contrário, apresentaram maior desejo em ter silhuetas maiores. Esses resultados são semelhantes aos obtidos em outros estudos que relatam que, independente do peso em que se encontram, as meninas, em geral, desejam ser mais magras, ou seja, diminuir a silhueta corporal, enquanto que os meninos almejam corpos mais fortes, logo silhuetas maiores ${ }^{30,33,34}$. Essa diferença entre os sexos pode ser explicada, dentre outros fatores, pela vulnerabilidade de ambos os sexos ao atual ideal de beleza que valoriza a magreza feminina e o corpo forte e musculoso masculi$\mathrm{no}^{8}$. Tais diferenças podem estar também relacionadas às influências culturais, através das quais as meninas, desde a infância até a vida adulta, são induzidas a praticarem atividades físicas que impliquem em perda de peso, com enfoque mais estético, enquanto os meninos são estimulados a praticarem atividades esportivas e outras competências sociais mais focadas na força física ${ }^{8}$. Em função destas diferenças, as estratégias que envolvem a melhor percepção e aceitação corporal entre adolescentes devem ser conduzidas de forma diferenciada para cada sexo.

Entre as variáveis investigadas para possíveis associações com a insatisfação com a imagem corporal e, comumente utilizadas no campo da saúde do adolescente e saúde coletiva, a exposição ao teasing relacionado ao corpo foi uma das que se mostraram mais fortemente associadas ao problema. Exposição ao teasing pode ser compreendida como a exposição a provocações ou como uma forma específica de assédio moral 
Tabela 2. Análise de regressão multinominal bivariada (Odds ratio e intervalo de confiança de 95\%) para a associação entre insatisfação com a imagem corporal e variáveis demográficas e socioeconômicas, antropométricas e de composição corporal, psicossocial e de estilo de vida.

\begin{tabular}{|c|c|c|c|c|c|c|c|c|c|}
\hline \multirow{3}{*}{ Variáveis } & \multirow{3}{*}{$\begin{array}{l}\text { Satisfeitos } \\
\quad(\%)\end{array}$} & \multicolumn{6}{|c|}{ Insatisfeitos } & \multirow[b]{3}{*}{ IC95\% } & \multirow[b]{3}{*}{$\begin{array}{l}\text { Valor } \\
\text { de p }\end{array}$} \\
\hline & & \multicolumn{3}{|c|}{$\begin{array}{c}\text { Desejam silhuetas } \\
\text { menores }\end{array}$} & \multicolumn{3}{|c|}{$\begin{array}{c}\text { Desejam silhuetas } \\
\text { maiores }\end{array}$} & & \\
\hline & & $\%$ & OR & IC95\% & $\begin{array}{l}\text { Valor } \\
\text { de p }\end{array}$ & $\%$ & OR & & \\
\hline \multicolumn{10}{|l|}{$\begin{array}{l}\text { II. Psicossocial e estilo } \\
\text { de vida }\end{array}$} \\
\hline \multicolumn{10}{|l|}{ Teasing } \\
\hline Sim & 9,7 & 57,2 & 4,79 & $3,00-7,65$ & $<0,01$ & 33,1 & 2,98 & $1,83-4,86$ & $<0,01$ \\
\hline Não & 29,8 & 36,4 & 1,0 & & & 33,8 & 1,0 & & \\
\hline \multicolumn{10}{|l|}{ Padrão de refeição } \\
\hline Insatisfatório & 20,1 & 51,7 & 2,70 & $1,96-3,74$ & $<0,01$ & 28,2 & 1,07 & $0,77-1,48$ & 0,68 \\
\hline Satisfatório & 30,7 & 29,1 & 1,0 & & & 40,2 & 1,0 & & \\
\hline \multicolumn{10}{|l|}{ Fumo } \\
\hline Sim & 42,9 & 35,7 & 0,49 & $0,21-1,15$ & 0,10 & 21,4 & 0,36 & $0,13-0,97$ & 0,04 \\
\hline Não & 24,4 & 41,6 & 1,0 & & & 34,0 & 1,0 & & \\
\hline \multicolumn{10}{|l|}{$\begin{array}{l}\text { Experimentação de } \\
\text { álcool }\end{array}$} \\
\hline Sim & 24,2 & 41,4 & 1,10 & $0,78-1,54$ & 0,60 & 34,4 & 1,19 & $0,83-1,70$ & 0,35 \\
\hline Não & 26,6 & 41,6 & 1,0 & & & 31,8 & 1,0 & & \\
\hline \multicolumn{10}{|l|}{ Consumo atual de álcool } \\
\hline Sim & 22,8 & 45,3 & 1,32 & $0,95-1,84$ & 0,09 & 31,9 & 1,06 & $0,75-1,49$ & 0,77 \\
\hline Não & 26,1 & 39,2 & 1,0 & & & 34,7 & 1,0 & & \\
\hline \multicolumn{10}{|l|}{ Atividade física } \\
\hline Pouco ativos & 28,4 & 46,3 & 1,04 & $0,48-2,26$ & 0,92 & 25,3 & 0,68 & $0,28-1,63$ & 0,39 \\
\hline Irregularmente ativos & 21,4 & 44,2 & 1,31 & $0,88-1,96$ & 0,18 & 34,4 & 1,22 & $0,80-1,85$ & 0,36 \\
\hline Muito ativos ou ativos & 25,7 & 40,4 & 1,0 & & & 33,9 & 1,0 & & \\
\hline \multicolumn{10}{|l|}{$\begin{array}{l}\text { Comportamento } \\
\text { sedentário }\end{array}$} \\
\hline Sim & 24,1 & 41,1 & 1,09 & $0,781-1,54$ & 0,59 & 34,8 & 1,26 & $0,88-1,80$ & 0,21 \\
\hline Não & 27,0 & 42,1 & 1,0 & & & 30,9 & 1,0 & & \\
\hline \multicolumn{10}{|l|}{$\begin{array}{l}\text { III. Antropométrica e } \\
\text { de composição corporal }\end{array}$} \\
\hline \multicolumn{10}{|l|}{ Classificação do IMC } \\
\hline Com excesso de peso & 14,3 & 82,3 & 6,22 & $4,18-9,26$ & $<0,01$ & 3,4 & 0,15 & $0,07-0,32$ & $<0,01$ \\
\hline Sem excesso de peso & 28,8 & 26,5 & 1,0 & & & 44,7 & 1,0 & & \\
\hline \multicolumn{10}{|l|}{$\begin{array}{l}\text { Perímetro da Cintura } \\
(\mathrm{cm})\end{array}$} \\
\hline$\geq \mathrm{P} 90$ & 4,5 & 92,8 & 15,70 & $5,95-41,6$ & $<0,01$ & 2,7 & 0,41 & $0,08-2,22$ & 0,30 \\
\hline$<\mathrm{P} 90$ & 27,3 & 35,5 & 1,0 & & & 37,2 & 1,0 & & \\
\hline \multicolumn{10}{|l|}{$\%$ de $\mathrm{GC}$} \\
\hline Elevado & 7,1 & 88,2 & 9,53 & $2,44-37,2$ & $<0,01$ & 4,7 & 0,46 & $0,09-2,47$ & 0,37 \\
\hline Não elevado & 26,2 & 38,0 & 1,0 & & & 35,8 & 1,0 & & \\
\hline
\end{tabular}

que sofrem os indivíduos que, de alguma forma, apresentam características físicas ou de comportamento que os diferenciam da maioria, sempre com uma conotação negativa ${ }^{35}$. Quando esta 
Tabela 3. Resultados da análise de regressão multinomial multivariada hierarquizada (Odds ratio e intervalo de confiança de 95\%) para associação entre insatisfação com a imagem corporal e variáveis demográficas e socioeconômicas, psicossocial, de estilo de vida, antropométricas e de composição corporal.

\begin{tabular}{|c|c|c|c|c|c|c|}
\hline \multirow{2}{*}{ Variáveis } & \multicolumn{3}{|c|}{$\begin{array}{c}\text { Desejam silhuetas } \\
\text { menores }\end{array}$} & \multicolumn{3}{|c|}{ Desejam silhuetas maiores } \\
\hline & OR & IC95\% & $\begin{array}{c}\text { valor } \\
\text { de p }\end{array}$ & OR & IC95\% & $\begin{array}{c}\text { valor } \\
\text { de p }\end{array}$ \\
\hline \multicolumn{7}{|c|}{ 1a Etapa - Variáveis demográficas e socioeconômicas } \\
\hline \multicolumn{7}{|l|}{ Sexo } \\
\hline Feminino & 2,00 & $1,45-2,77$ & $<0,0$ & 0,77 & $0,56-1,08$ & 0,13 \\
\hline Masculino & 1,0 & & & 1,0 & & \\
\hline \multicolumn{7}{|l|}{ Faixa etária } \\
\hline$\geq 15$ anos & 0,85 & $0,56-1,30$ & 0,46 & 1,10 & $0,69-1,75$ & 0,69 \\
\hline$<15$ anos & 1,0 & & & 1,0 & & \\
\hline \multicolumn{7}{|l|}{ Cor } \\
\hline Preto/pardo/amarelo/indígena & 0,92 & $0,66-1,27$ & 0,61 & 1,13 & $0,81-1,59$ & 0,47 \\
\hline Branco & 1,0 & & & 1,0 & & \\
\hline \multicolumn{7}{|l|}{ Tipo de escola } \\
\hline Particular & 1,51 & $1,00-2,28$ & 0,05 & 0,85 & $0,56-1,29$ & 0,45 \\
\hline Pública & 1,0 & & & 1,0 & & \\
\hline \multicolumn{7}{|l|}{ Classificação social e econômica } \\
\hline A & 1,44 & $0,76-2,75$ & 0,26 & 1,07 & $0,56-2,07$ & 0,84 \\
\hline $\mathrm{B}$ & 0,93 & $0,57-1,51$ & 0,76 & 0,74 & $0,47-1,16$ & 0,19 \\
\hline C e D & 1,0 & & & 1,0 & & \\
\hline \multicolumn{7}{|l|}{ Escolaridade do chefe de família } \\
\hline 9 a 12 anos & 0,57 & $0,31-1,05$ & 0,07 & 1,03 & $0,54-1,98$ & 0,92 \\
\hline 5 a 8 anos & 1,16 & $0,61-2,23$ & 0,65 & 1,48 & $0,74-2,97$ & 0,27 \\
\hline 0 a 4 anos & 1,0 & & & 1,0 & & \\
\hline \multicolumn{7}{|l|}{ 2a Etapa - Variável psicossocial } \\
\hline \multicolumn{7}{|l|}{ Sexo } \\
\hline Feminino & 1,95 & $1,41-2,70$ & $<0,01$ & 0,76 & $0,55-1,05$ & 0,11 \\
\hline Masculino & 1,0 & & & 1,0 & & \\
\hline \multicolumn{7}{|l|}{ Tipo de escola } \\
\hline Particular & 1,32 & $0,96-1,83$ & 0,09 & 0,73 & $0,53-1,01$ & 0,06 \\
\hline Pública & 1,0 & & & 1,0 & & \\
\hline \multicolumn{7}{|l|}{ Teasing } \\
\hline Sim & 4,70 & $2,94-7,51$ & $<0,01$ & 3,00 & $1,84-4,91$ & $<0,01$ \\
\hline Não & 1,0 & & & 1,0 & & \\
\hline
\end{tabular}

Além de se constituir uma forma de violência, em geral entre pares, estudos mostram que os adolescentes que sofrem teasing pela sua forma corporal apresentam menor propensão à adesão a comportamentos saudáveis como consumo de frutas, verduras e legumes e prática de atividade física, e uma maior propensão a comportamentos inadequados para controle de peso, como práticas purgativas e dietas restritivas, quando comparados àquelas que não sofreram tal exposição ${ }^{19,30}$. Tais práticas inadequadas para controle de peso tendem a aumentar quando o adolescen- te sofre exposição ao teasing, não só na escola, mas também na família ${ }^{30}$. Desta forma, para que as ações de enfrentamento deste tipo de problema sejam efetivas, é preciso que ultrapassem o ambiente escolar e cheguem às famílias.

Corroborando estudos anteriores ${ }^{36}$ adolescentes com padrão de refeição insatisfatório apresentaram maiores prevalências de insatisfação corporal, particularmente para o desejo de ter silhuetas menores, em relação aos que apresentaram padrão satisfatório. Essas evidências sugerem que os adolescentes podem omitir refei- 
Tabela 3. Resultados da análise de regressão multinomial multivariada hierarquizada (Odds ratio e intervalo de confiança de 95\%) para associação entre insatisfação com a imagem corporal e variáveis demográficas e socioeconômicas, psicossocial, de estilo de vida, antropométricas e de composição corporal.

\begin{tabular}{|c|c|c|c|c|c|c|}
\hline \multirow{2}{*}{ Variáveis } & \multicolumn{3}{|c|}{$\begin{array}{c}\text { Desejam silhuetas } \\
\text { menores }\end{array}$} & \multicolumn{3}{|c|}{ Desejam silhuetas maiores } \\
\hline & OR & IC95\% & $\begin{array}{c}\text { valor } \\
\text { de p }\end{array}$ & OR & IC95\% & $\begin{array}{l}\text { valor } \\
\text { de p }\end{array}$ \\
\hline \multicolumn{7}{|c|}{ 3a Etapa - Variáveis relacionadas ao estilo de vida } \\
\hline \multicolumn{7}{|l|}{ Sexo } \\
\hline Feminino & 1,71 & $1,22-2,40$ & $<0,01$ & 0,76 & $0,54-1,07$ & 0,11 \\
\hline Masculino & 1,0 & & & 1,0 & & \\
\hline \multicolumn{7}{|l|}{ Teasing } \\
\hline Sim & 4,55 & $2,83-7,33$ & $<0,01$ & 3,00 & $1,84-4,91$ & $<0,01$ \\
\hline Não & 1,0 & & & 1,0 & & \\
\hline \multicolumn{7}{|l|}{ Padrão de refeição } \\
\hline Insatisfatório & 2,50 & $1,78-3,51$ & $<0,01$ & 1,10 & $0,78-1,54$ & 0,59 \\
\hline Satisfatório & 1,0 & & & 1,0 & & \\
\hline \multicolumn{7}{|l|}{ Fumo } \\
\hline Sim & 0,36 & $0,14-0,90$ & 0,03 & 0,34 & $0,12-0,96$ & 0,04 \\
\hline Não & 1,0 & & & 1,0 & & \\
\hline \multicolumn{7}{|l|}{ Experimentação de álcool } \\
\hline Sim & 0,85 & $0,56-1,28$ & 0,44 & 1,18 & $0,78-1,78$ & 0,43 \\
\hline Não & 1,0 & & & 1,0 & & \\
\hline \multicolumn{7}{|l|}{ Consumo atual de álcool } \\
\hline Sim & 1,42 & $0,95-2,12$ & 0,09 & 1,06 & $0,70-1,58$ & 0,80 \\
\hline Não & 1,0 & & & 1,0 & & \\
\hline \multicolumn{7}{|l|}{ Atividade física } \\
\hline Pouco ativos & 0,99 & $0,44-2,21$ & 0,98 & 0,76 & $0,31-1,87$ & 0,56 \\
\hline Irregularmente ativos & 1,18 & $0,77-1,81$ & 0,45 & 1,29 & $0,84-1,99$ & 0,25 \\
\hline Muito ativos ou ativos & 1,0 & & & 1,0 & & \\
\hline \multicolumn{7}{|l|}{ Comportamento sedentário } \\
\hline Sim & 1,10 & $0,77-1,57$ & 0,62 & 1,22 & $0,84-1,76$ & 0,30 \\
\hline Não & 1,0 & & & 1,0 & & \\
\hline \multicolumn{7}{|c|}{$\begin{array}{l}4^{\mathrm{a}} \text { Etapa (modelo final) - Variáveis antropométricas e } \\
\text { de composição corporal }\end{array}$} \\
\hline \multicolumn{7}{|l|}{ Sexo } \\
\hline Feminino & 2,58 & $1,77-3,78$ & $<0,01$ & 0,65 & $0,46-0,92$ & $<0,01$ \\
\hline Masculino & 1,0 & & & 1,0 & & \\
\hline \multicolumn{7}{|l|}{ Teasing } \\
\hline $\operatorname{Sim}$ & 3,15 & $1,90-5,22$ & $<0,01$ & 3,41 & $2,06-5,64$ & $<0,01$ \\
\hline Não & 1,0 & & & 1,0 & & \\
\hline \multicolumn{7}{|l|}{ Padrão de refeição } \\
\hline Insatisfatório & 2,41 & $1,68-3,47$ & $<0,01$ & 1,09 & $0,77-1,53$ & 0,62 \\
\hline Satisfatório & 1,0 & & & 1,0 & & \\
\hline \multicolumn{7}{|l|}{ Fumo } \\
\hline Sim & 0,36 & $0,13-0,98$ & 0,05 & 0,37 & $0,13-1,05$ & 0,06 \\
\hline Não & 1,0 & & & 1,0 & & \\
\hline \multicolumn{7}{|l|}{ Classificação com base no IMC } \\
\hline Com excesso de peso & 5,08 & $3,19-8,08$ & $<0,01$ & 0,12 & $0,05-0,27$ & $<0,01$ \\
\hline Sem excesso de peso & 1,0 & & & 1,0 & & \\
\hline \multicolumn{7}{|l|}{ Perímetro da Cintura } \\
\hline$\geq \mathrm{P} 90$ & 3,71 & $1,03-13,42$ & 0,05 & 1,41 & $0,20-10,07$ & 0,73 \\
\hline$<\mathrm{P} 90$ & 1,0 & & & 1,0 & & \\
\hline \multicolumn{7}{|l|}{$\%$ de $\mathrm{GC}^{\mathrm{t}}$} \\
\hline Elevado & 1,64 & $0,31-8,52$ & 0,56 & 0,78 & $0,07-9,24$ & 0,84 \\
\hline Não elevado & 1,0 & & & 1,0 & & \\
\hline
\end{tabular}

${ }^{\varepsilon}$ Indice de massa corporal (peso/estatura ${ }^{2}$ ); ${ }^{\mathrm{t}}$ Gordura corporal. 
ções como estratégia inadequada para perda de peso, independentemente de ter ou não excesso de peso, já que a associação se manteve mesmo após o ajuste para adequação de peso, justificando a necessidade de desmistificação desta prática.

Os adolescentes fumantes apresentaram uma menor chance para o desejo de ter silhuetas menores. O motivo que poderia justificar tal associação seria o fato dos fumantes, normalmente, apresentarem o medo de engordar, caso parem de fumar, e talvez utilizem o cigarro como método de controle de peso ${ }^{37}$. Por outro lado, Duca et al. ${ }^{33}$, que investigaram a mesma relação, não observaram associação entre estas variáveis.

Como esperado, o excesso de peso corporal esteve associado com o desejo por uma silhueta menor, corroborando com estudos avaliados em recente revisão sistemática de literatura ${ }^{5}$. Da mesma forma, o perímetro da cintura elevado também esteve associado ao desejo por uma silhueta menor. Estes resultados expressam uma percepção realista dos indivíduos que tem medidas corporais elevadas o que, de certa forma, favorece as intervenções que buscam adequação de tais medidas com a perspectiva de melhor estado de saúde. Já o percentual de gordura corporal elevado não se mostrou associado à insatisfação com a silhueta corporal na análise multivariada. Tais achados podem estar indicando que o IMC e perímetro da cintura podem refletir melhor a percepção do adolescente quanto às suas formas corporais do que o percentual de gordura.

O presente estudo apresenta algumas limitações. A amostra do estudo não é representativa de uma determinada área geográfica ou grupo populacional específico, pois compreendeu adolescentes de quatro escolas privadas e duas escolas públicas da região metropolitana do Rio de Janeiro. Desta forma, a generalização dos achados relativos à prevalência da insatisfação com a imagem corporal para uma população mais abrangente de adolescentes deve ser evitada. Uma segunda limitação importante é o fato de termos um delineamento transversal que estima as associações entre as variáveis em um único momento do tempo, não permitindo estabelecer relações de causa e efeito entre as variáveis de interesse. Uma terceira limitação seria a utilização da Escala de Silhuetas Corporais para a aferição da insa- tisfação com imagem corporal. Segundo Gardner et al. ${ }^{38}$, a quantidade e a semelhança das silhuetas apresentadas poderiam confundir o indivíduo na identificação entre a sua imagem considerada atual e a que desejaria ter. Todavia, a Escala de Silhuetas tem sido amplamente utilizada por outros autores para avaliação da percepção corporal em função de vantagens operacionais, tais como, a facilidade de aplicação em estudos clínicos e epidemiológicos e o baixo custo ${ }^{30,39}$.

Como pontos positivos da pesquisa apontamos, primeiramente, o fato de ter utilizado uma abordagem multivariada hierárquica no processo de análise de dados que permite, por meio de uma perspectiva multidimensional, identificar subgrupos vulneráveis à insatisfação com a imagem corporal. A possibilidade de investigar tanto a insatisfação com o desejo de silhuetas menores, como a insatisfação com o desejo de silhuetas maiores do que as silhuetas atuais percebidas também foi um ponto forte do estudo, pois permitiu identificar subgrupos de risco diferenciados, de acordo com cada um dos tipos de insatisfação.

Como apontado ao longo do texto, as meninas, aqueles com excesso de peso, circunferência da cintura elevada e com padrão de realização de refeições insatisfatório foram os subgrupos mais vulneráveis ao desejo de ter silhueta menor do que a atual autopercebida, enquanto que os meninos e aqueles sem excesso de peso parecem ser mais vulneráveis ao desejo de ter silhuetas maiores. Por outro lado, aqueles expostos ao teasing relacionado ao corpo são mais vulneráveis, tanto à insatisfação corporal pelo desejo de ter silhuetas menores, como por desejar silhuetas maiores. Este perfil diferenciado em termos de insatisfação ressalta a importância de se incorporar aspectos específicos às ações de promoção de saúde nas escolas e em outros ambientes de socialização de adolescentes e jovens, que levem em consideração as diferenças culturais de gênero e demais aspectos aqui investigados. Também parece de extrema relevância que políticas públicas e ações visando uma percepção mais positiva da autoimagem e melhor aceitação das diferenças estéticas entre os adolescentes sejam estimuladas, não se restringindo ao espaço escolar, mas que também envolvam as famílias e outros espaços sociais. 


\section{Colaboradores}

GX Carvalho foi responsável pela revisão de literatura, pela análise dos dados e redação principal do texto. APN Nunes colaborou nas análises dos dados e revisão do texto. CL Moraes participou da concepção do estudo e revisão do texto. GV Veiga foi responsável pela concepção do estudo de base ELANA e do presente estudo, pela coordenação de todo trabalho de campo e pela revisão do texto.

\section{Agradecimentos}

Ao Conselho Nacional de Desenvolvimento Científico e Tecnológico (CNPq) e à Fundação Carlos Chagas Filho de Amparo à Pesquisa do Estado do Rio de Janeiro (FAPERJ) pelo financiamento do ELANA, e à Coordenação de Aperfeiçoamento de Pessoal de Nível Superior (CAPES) pela bolsa concedida a autora principal para realização do curso de Mestrado.

\section{Referências}

1. Dion J, Blackburn ME, Auclair J, Laberge L, Veillette S, Gaudreault M, Touchette E. Development and aetiology of body dissatisfaction in adolescent boys and girls. Int J Adolesc Youth 2015; 20(2):151-166.

2. Alves D, Pinto M, Alves S, Mota A, Leirós V. Cultura e imagem corporal. Motricidade 2009; 5(1):1-20.

3. Castro IRRD, Levy RB, Cardoso LDO, Passos MDD, Sardinha LMV, Tavares LF, Martins A, Castro IRR. Imagem corporal, estado nutricional e comportamento com relação ao peso entre adolescentes brasileiros. Cien Saude Colet 2010; 15(2):3099-4108.

4. Poli Neto P, Caponi SNC. A medicalização da beleza. Interface (Botucatu) 2007; 11(23):569-584.

5. Jiménez-Flores P, Jiménez-Cruz A, Bacardí-Gascón M. Body-image dissatisfaction in children and adolescents: a systematic review. Nutr Hosp 2017; 34(2):479 489.

6. Levandoski G, Cardoso FL. Imagem corporal e status social de estudantes brasileiros envolvidos em bullying. Rev Latinoam Psicol 2013; 45(1):135-145.

7. Shirasawa T, Ochiai H, Nanri H, Nishimura R, Ohtsu T, Hoshino, H, Kokaze A. The relationship between distorted body image and lifestyle among Japanese adolescents: a population-based study. Arch Public Health 2015; 73(32):1-7.

8. Braga PD, Molina MDCB, Figueiredo TAM. Representações do corpo: com a palavra um grupo de adolescentes de classes populares. Cien Saude Colet 2010; 15(1):87-95.

9. Santos JFS, Oliveira P, Campos AA, Pereira EO, Sousa EA. Relação entre Insatisfação Corporal e Atividade Física em Adolescentes da Zona Urbana de Irati-PR. Rev Educ Fís/UEM 2014; 25(2):193-201

10. Pelegrini A. Coqueiro RS, Beck CC, Ghedin KD, Lopes AS, Petroski EL. Dissatisfaction with body image among adolescent students: association with sociodemographic factors and nutritional status. Cien Saude Colet 2014; 19(4):1201-1208.

11. McElhone S, Kearney JM, Giachetti I, Zunft HJF, Martínez JA. Body image perception in relation to recent changes and strategies for weight loss in a nationally representative sample in the European Union. Public Health Nutr 1999; 2(1a):143-151.

12. Madrigal-Fritsch H, Irala-Estévez JD, Martínez-González MA, Kearney J, Gibney M, Martínez-Hernández JA. Percepción de la imagen corporal como aproximación cualitativa al estado de nutrición. Salud Pública Mex 1999; 41(6):479-486.

13. Instituto Brasileiro de Geografia e Estatística (IBGE). Características étnico-raciais da população. Um estudo das categorias de classificação de cor ou raça. Rio de Janeiro: IBGE; 2008.

14. Lepsen AM, Silva MZ. Prevalência e fatores associados à insatisfação com imagem corporal de adolescentes de escolas do Ensino Médio da zona rural da região sul do Rio Grande do Sul 2012. Epidemiol Serv Saúde Brasília 2014; 23(2):317-325.

15. Associação Brasileira de Empresas de Pesquisas (ABEP). Codes and Guides: Brazilian Economic Classification Criterion. São Paulo: ABEP; 2008. 
16. Estima CC, Costa RS, Sichieri R, Pereira RA, Veiga GV. Meal consumption patterns and anthropometric measurements in adolescents from a low socioeconomic neighborhood in the metropolitan area of Rio de Janeiro, Brazil. Appetite 2009; 52(3):735-739.

17. Guedes DP, Lopes CC, Guedes JERP. Reprodutibilidade e validade do Questionário Internacional de Atividade Física em adolescentes. Rev Bras Med Esporte 2005; 11(2):151-158.

18. Matsudo SM, Matsudo VK, Araújo T, Andrade D, Andrade E, Oliveira L, Braggion G. Nível de atividade física da população do Estado de São Paulo: análise de acordo com o gênero, idade, nível sócio-econômico, distribuição geográfica e de conhecimento. Rev Bras Cien Mov 2008; 10(4):41-50.

19. World Health Organization (WHO). Inequalities young people's health: key findings from the Health Behaviour in School-aged Children (HBSC) 2005/2006 survey fact sheet. Copenhagen: WHO; 2008.

20. Vessey JA, Horowitz JA, Carlson KL, Duffy M. Psychometric evaluation of the child-adolescent teasing scale. J Sch Health 2008; 78(6):344-350.

21. Leme ACB, Philippi ST. Provocações e comportamentos para controle de peso em adolescentes do sexo feminino. Rev Paul Pediatria 2013; 31(4):431-436.

22. Menzel JE, Schaefer LM, Burke NL, Mayhew LL, Brannick MT, Thompson JK. Appearance-related teasing, body dissatisfaction, and disordered eating: a meta-analysis. Body Image 2010; 7(4):261-270.

23. Habicht JP. Estandarizacion de metodos epidemiológicos cuantitativos sobre el terreno. Bol Sanit Panam 1974; 76(5):375-384.

24. Onis MD, Onyango AW, Borghi E, Siyam A, Nishida C, Siekmann J. Development of a WHO growth reference for school-aged children and adolescents. Bull World Health Org 2007; 85(9):660-667.

25. Houtkooper LB, Going SB, Lohman TG, Roche AF, Van Loan M. Bioelectrical impedance estimation of fat-free body mass in children and youth: A cross-validation study. J Appl Physiol 1992; 72(1)366-373.

26. Williams DP, Going SB, Lohman TG, Harsha DW, Srinivasan SR, Webber LS, Berenson GS. Body fatness and risk for elevated blood pressure total cholesterol and serum lipoprotein ratios in children and adolescents. Am J Public Health 1992; 82(3):358-363.

27. DB. Inference and missing data. Biometrika 1976; 63(3):581-592.

28. Nunes LN. Métodos de imputação de dados aplicados na área da saúde [dissertação]. Rio Grande do Sul: Universidade Federal do Rio Grande do Sul; 2007.

29. Víctora CG, Huttly SR, Fuchs SC, Olinto MT. The role of conceptual frameworks in epidemiological analysis: a hierarchical approach. Int J Epidemiol 1997; 26(1):224-227.
30. Petroski EL, Pelegrini A, Glaner MF. Motivos e prevalência de insatisfação com a imagem corporal em adolescentes. Cien Saude Colet 2012; 17(4):1071-1077.

31. Cheah WL, Hazmi H, Chang CT. Disordered eating and body image issues and their associated factors among adolescents in urban secondary schools in Sarawak, Malaysia. Int J Adolesc Med Health 2017; 29(2):20150044.

32. Palma A, Resende F, Sant'anna MR, Assis M, Teves N, Moreira JPL. Insatisfação com o peso e a massa corporal em estudantes do ensino fundamental e médio do sexo feminino no município do Rio de Janeiro. Rev Bras Cienc Esporte 2013; 35(1):51-64.

33. Duca GF, Garcia LMT, Sousa TD, Oliveira ED, Nahas MV. Insatisfação com o peso corporal e fatores associados em adolescentes. Rev Paul Pediatr 2010; 28(4):340-346.

34. Holsen I, Jones DC, Birkeland MS. Body image satisfaction among Norwegian adolescents and young adults: A longitudinal study of the influence of interpersonal relationships and BMI. Body Image 2012; 9(2):201-208.

35. Neumark-Sztainer D, Wall M, Story M, Standish, AR . Dieting and unhealthy weight control behaviors during adolescence: Do they predict changes in weight ten years later? J Adolesc Health 2012; 50(1):80-86.

36. Bibiloni MM, Pich J, Pons A, Tur JA. Body image and eating patterns among adolescents. BMC Public Health 2013; 13:1104.

37. Winter AL, Guia NA, Ferrence R, Cohen JE. The relationship between body weight perceptions, weight control behaviours and smoking status 121 among adolescents. Can J Public Health 2001; 93(5):362-365.

38. Gardner RM, Friedman BN, Jackson NA. Methodological concerns when using silhouettes to measure body image. Percept Mot Skills 1998; 86(2):387-395.

39. Pelegrini A, Silva DAS, Silva AF, Petroski EL. Insatisfação corporal associada a indicadores antropométricos em adolescentes de um cidade com índice de desenvolvimento humano médio a baixo. Rev Bras Cienc Esporte 2011; 33(3):687-698.

Artigo apresentado em 25/03/2018

Aprovado em 26/11/2018

Versão final apresentada em 28/11/2018 